Research Paper

\title{
Reprogramming Factors Remodel Melanoma Cell Phenotype by Changing Stat3 Expression
}

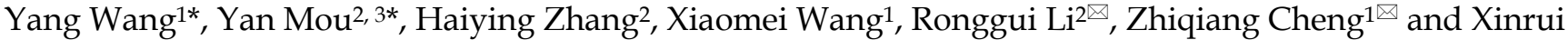 \\ $\mathrm{Liu}^{4 凶}$ \\ 1. Department of Pathology, Shenzhen People's Hospital, Second Clinical Medical College of Jinan University, Shenzhen, Guangdong, China; \\ 2. Key Laboratory of Pathobiology, Ministry of Education, Norman Bethune College of Medicine, Jilin University, Changchun, China; \\ 3. The Second Hospital of Jilin University, Changchun, China; \\ 4. Jilin Academy of Traditional Chinese Medicine, Changchun, China. \\ * These authors contributed equally to this work
}

$\triangle$ Corresponding authors: Dr. Ronggui Li, The Key Laboratory of Pathobiology, Ministry of Education, Norman Bethune College of Medicine, Jilin University, Changchun, 130021, P.R. China. Tel.: 86-431 85619481; E-mail: lirg@jlu.edu.cn; Dr. Zhiqiang Cheng, Department of Pathology, Shenzhen People's Hospital, Second Clinical Medical College of Jinan University, Shenzhen, Guangdong, 518020, P.R. China. Tel.: 86-755 22948392; E-mail: chengzhiqiang2004@aliyun.com; and Dr. Xinrui Liu, Jilin Academy of Traditional Chinese Medicine, 1745 Works and Peasants Road, Changchun, 130021, P.R. China. Tel.: 86-431 86816453; E-mail: 2908081176@qq.com.

(c) Ivyspring International Publisher. This is an open access article distributed under the terms of the Creative Commons Attribution (CC BY-NC) license (https://creativecommons.org/licenses/by-nc/4.0/). See http://ivyspring.com/terms for full terms and conditions.

Received: 2017.07.17; Accepted: 2017.10.11; Published: 2017.11.02

\begin{abstract}
The limited availability of melanoma stem cells is a major challenge for therapeutic reagent screening and study of molecular mechanisms. It has been shown that induced expression of four stem cell factors (Oct4, Sox2, KIf4, and c-Myc) changes the phenotype of osteosarcoma and breast cancer cells to osteosarcoma stem cells and breast cancer stem cells, respectively. The present study aimed to explore whether these four factors might change the phenotype of melanoma cells to melanoma stem cells and, if so, to examine the possible molecular signal involved. Melanoma B16-F10 cells were transfected with the plasmid TetO-FUW-OSKM which contains cDNA expressing four factors, driven by the Tet-On element. We found that expression of the four transcription factors was highly induced by DOX in the stable melanoma cell clones. Further studies confirmed that induced expression of these factors remodeled the phenotype of the melanoma cells to melanoma stem cells (MSCs). This conclusion was supported by the evidence that induced expression of these factors increased the numbers of tumor-initiating cells, (namely MSCs), both in an in vitro cell culture system and in a mouse in vivo model. The conclusion was further supported by the observation that the induction of these factors exclusively increased the mRNA of signal transducer and activator of transcription 3 which has been reported to play a crucial role in stem cell maintenance. Thus, phenotypic remodeling of melanoma cells following the induction of these four factors provided a simple and optimal means to constantly obtain MSCs for screening new therapeutic reagents. The result also reveals that Stat 3 may be a crucial link between the induction of the four factors and the cell remodeling, suggesting its potential role as a target to fight melanoma.
\end{abstract}

Key words: Phenotypic Remodeling; Melanoma; Transcription Factors; Stat3; Melanoma Stem Cells.

\section{Introduction}

Malignant melanoma is a deadly disease with highly aggressive and drug-resistant behavior [1]. The incidence of melanoma is higher in children and adolescents who may have a long life expectancy [2]. Conventional surgery, chemotherapy, radiotherapy and biotherapy are not always successful and some of these treatments are associated with adverse reactions and/or emergence of drug resistance $[3,4]$, because of relatively poor understanding of the cellular and molecular mechanisms involved.

Accumulated evidence has shown that a small subpopulation of malignant cells has stem cell properties, is responsible for tumor recurrence, and so named tumor-initiating cells (TICs) or cancer stem 
cells (CSCs) [5]. The resistance of CSCs to currently-used chemical reagents may be a major reason for the treatment failure [6]. Although these reagents may be able to eliminate the bulk of the neoplasm and decrease the size of the tumors, they are unable to eliminate the CSCs which are responsible for tumor relapse and drug resistance. Consequently, specific therapy aimed at CSCs might provide promise for malignant melanoma treatment.

The scarce availability of CSCs is a major challenge for therapeutic reagent screening. Until recently, CSCs have been identified in leukemia and some solid tumors such as colon and breast cancers and some malignant brain tumors. Preliminary studies have shown that chemical reagents aimed at these CSCs have better therapeutic effects [7, 8]. Nevertheless, the number of native CSCs is far less than the requirement for broad studies.

Accumulating evidence has shown that among melanoma cells, there is a subpopulation with a tumor-initiating phenotype, named melanoma stem-like cells (MSCs) [9, 10]. Moreover, some molecular markers of MSCs have been explored [11-14]. However, the limited availability of MSCs prevents their broad application in molecular mechanism research and therapeutic drug screening. Yamanaka's four transcription factors: Oct4, Sox2, Klf4, and c-Myc (OSKM) have been successfully used to induce osteosarcoma cells and breast cancer cells into osteosarcoma stem cells and breast cancer stem cells, respectively [15-18]. In the present study, the plasmid expressing these four factors driven by the Tet-On element was transfected into melanoma F10-B16 cells and doxycycline (DOX) was used to induce the expression of these factors in stable transfected cell clones. The effects of induced expression of these factors on the phenotype of melanoma cells and involved molecular signals were studied.

\section{Materials and Methods}

\section{Materials}

High Glucose Dulbecco's Modified Eagle Medium (H-DMEM) and sucrose-based solution were from Gibco BRL (Rockville, MD, USA). Fetal bovine serum (FBS) was from HyClone Inc. (Logan, UT, USA). Trizol Reagent, PCR primers, and RT-reaction Kit were purchased from TaKaRa Biotechnology (Dalian, Liaoning, China). SYBR Green PCR Master Mix was from Applied Biosystems (Warrington, UK). LipofectamineTM2000 and Zeosin were from Invitrogen (Carlsbad, CA, USA). Basic fibroblastic growth factor (bFGF) was from Gibco (California, USA). C57BL/ 6 mice were from Jilin University (Jilin,
China). Doxycycline (DOX) was from Sigma (San Francisco, USA). Plasmids TetO-FUW-OSKM and FUW-M2rtTA were gifts from Rudolf Jaenisch (Addgene plasmid \# 20321 and \# 20342).

\section{Cell culture and transfection}

B16-F10 melanoma cells were from ATCC (Maryland, USA). The cells were maintained in H-DMEM supplemented with $10 \%$ FBS at $37 \mathrm{C}$ in $5 \%$ $\mathrm{CO} 2$ and a humidified atmosphere. All the transfection was performed using LipofectAMINE 2000 reagent, according to the manufacturer's instructions. After 24h, the transfection was ended by washing away old media and adding fresh complete media to the cells. We split the cells in a $10-\mathrm{cm}$ plate at day 3 and added Zeocin $(400 \mu \mathrm{g} / \mathrm{ml})$ at day 5 to start the selection. The cells were checked every day and the medium was changed when necessary to remove the dead cells. When the clones were clearly visible and isolated from one another, they were picked, transferred to 96-well plates, and then expanded.

\section{RNA purification and RT-QPCR}

Total RNA purification and RT-QPCR were performed with a detailed procedure as described previously [19]. The primer sets used for PCR amplification are shown in Table 1. After amplification, a melting curve was generated and data analysis was performed by using Dissociation Curves 1.0 software (Applied Biosystems). The normalized value was given by the ratio of mRNA of the target gene to mRNA of the reference gene ( $\beta$-actin) in each sample.

\section{Immunocytochemical staining}

Following the manufacturer's instruction, immunocytochemical staining was carried out by a SP DAB Detection Kit, based on Streptavidin-Peroxidase, purchased from Fuzhou Maixin Biotech Co. Ltd (Fuzhou, China). Cells were stained on primary rabbit anti-Oct4 and c-Myc polyclonal antibodies (Abcam, Cambridge, UK) after quenching endogenous peroxidase activity for $30 \mathrm{~min}$ at room temperature. Positive immunochemical reactions were, then detected by anti-rabbit secondary (30 $\mathrm{min}$ at room temperature) and revealed using $\mathrm{DAB}$ chromagen. The stained slides were counterstained with hematoxylin.

\section{Western blotting}

Protein samples were resolved by $10 \%$ SDS-PAGE and then were transferred onto polyvinylidene fluoride (PVDF) membranes (Millipore, Bedford, USA). After blocking and washing, the membranes were incubated with primary rabbit anti-Oct4 and c-Myc polyclonal 
antibodies and mouse anti- $\beta$-actin monoclonal antibody (Abcam, Cambridge, UK). Following extensive washing, the membranes were incubated with HRP-labeled anti-rabbit IgG or HRP-labeled anti-mouse IgG for $1 \mathrm{~h}$. After washing the membranes, the immunoreactive bands were visualized by using an ECL kit, and then the membranes were exposed to film and analyzed by using an automatic gel imaging analysis system (Gene, Cambridge, UK).

Table 1. Primer sets used for RT-QPCR

\begin{tabular}{|c|c|c|c|}
\hline Genes & $\begin{array}{l}\text { Primer } \\
\text { sets }\end{array}$ & Sequences & GenBank \\
\hline \multirow[t]{2}{*}{ Oct4 } & Forward & 5'-CAGCCAGACCACCATCTGTC-3' & NM_01363 \\
\hline & Reverse & 5'-GTCTCCGATTTGCATATCTCCTG-3' & \\
\hline \multirow[t]{2}{*}{ Sox2 } & Forward & 5'-GCTCGCAGACCTACATGAAC-3' & NM_011443 \\
\hline & Reverse & 5'-GCCTCGGACTTGACCACAG-3' & \\
\hline \multirow[t]{2}{*}{ Klf4 } & Forward & 5'-CTTCAGCTATCCGATCCGGG-3' & NM_010637 \\
\hline & Reverse & 5'-GAGGGGCTCACGTCATTGAT-3' & \\
\hline \multirow[t]{2}{*}{ c-Myc } & Forward & 5'-TCTCCATCCTATGTTGCGGTC-3' & NM_010849 \\
\hline & Reverse & 5'-TCCAAGTAACTCGGTCATCATCT-3' & \\
\hline \multirow[t]{2}{*}{ Stat3 } & Forward & 5'- GGATCGTGGCCCGATGCCTG -3' & NM_011486.5 \\
\hline & Reverse & 5'- CTACGGCGGCTGTTGGGTGG -3' & \\
\hline \multirow{2}{*}{$\begin{array}{l}\text { Cyclin } \\
\text { A1 }\end{array}$} & Forward & 5'- CCCATCGACCGCAGCAAGCA -3' & NM_007628.3 \\
\hline & Reverse & 5'- AGGCGGCTCCATGAGGGACA -3' & \\
\hline \multirow[t]{2}{*}{ CDK1 } & Forward & 5'- GTTGCTGGGCTCGGCTCGTT -3' & NM_007659 \\
\hline & Reverse & 5'- GCGGCTTCTTGGTGGCCAGT -3' & \\
\hline \multirow[t]{2}{*}{ SKP2 } & Forward & 5'- TGCCCCAACCTCATCCGCCT -3' & NM_013787 \\
\hline & Reverse & 5'- ACCGGCTGAGCGAGAGGTGT -3' & \\
\hline \multirow[t]{2}{*}{ P53 } & Forward & 5'-GGACGATCTGTTGCTGCCCCGAGA-3' & NM_011640 \\
\hline & Reverse & 5'- TGACAGGGGCCATGGAGTGGCT -3' & \\
\hline \multirow[t]{2}{*}{$\beta$-actin } & Forward & 5'-CATGTACGTTGCTATCCAGGC-3' & NM_001101 \\
\hline & Reverse & 5'-CTCCTTAATGTCACGCACGAT-3' & \\
\hline
\end{tabular}

\section{Sphere formation efficiency (SFE) assay}

Melanoma sphere formation efficiency assay was performed with a detailed procedure as described previously [20]. Briefly, cells were planted at a density of 1000 cells in 24-well ultra low attachment plates (Corning, Inc.) in H-DMEM with 5\% FBS and bFGF (20ng/ $\mathrm{ml})$. Fresh aliquots of bFGF were added every 2 days. After 2 weeks in culture, the cell spheres, whose diameters are greater than 50 micrometer, were counted under inverted phase contrast microscopy.

\section{Tumor formation}

Two groups of 10 C57BL/ 6 female mice aged 8 to 10 weeks, with an average weight of $20 \mathrm{~g}$ were injected subcutaneously with one thousand of B16-F10 melanoma cells, suspended in 100 1 PBS. Drinking water containing $2 \%$ sucrose, with or without addition of DOX $(5 \mathrm{mg} / \mathrm{ml})$, was given to the induced and control mice, respectively. Mice were sacrificed after 2 weeks and the tumors were detached. The weights and the diameters of the tumors were measured. The longest and the shortest diameters of each tumor were named " $a$ " and " $b$ ", respectively. The volumes were calculated according to the formula volumes $=\left(4 \times \Pi \times \mathrm{R}^{3}\right) / 3$, in which $\mathrm{R}=(\mathrm{a}$ + b) $/ 2$.

\section{Statistical analysis}

All calculations and statistical analyses were performed by using GraphPad Prism 5.0 software (San Diego, CA, USA). Student's t test was used to analyze the significance of any differences between two groups. $\mathrm{P}<0.05$ was considered statistically significant.

\section{Results}

\section{Induction of OSKM by DOX}

To identify the cell clones in which the expressions of four factors (OSKM) had been induced by the exposure to DOX, the mRNAs of OSKM were analyzed by RT-QPCR in three stable cell clones which had been transfected with the vector in which the transcription of the four factors was controlled by the Tet-on system. The results are shown in Fig. 1. In all three tested cell clones OSKM mRNAs were highly induced by DOX treatment, compared to the cells without exposure to DOX and the parental melanoma cells without transfection (Fig. 1A). We also analyzed the expression of both Oct4 and c-Myc protein in one cell clone (1\#) by immunochemistry and western blot. Fig. 1B and Fig. 1C show representative results of the immunochemistry staining and western blot, respectively. Fig. 1D shows the results of statistical analysis. The high induction of both Oct 4 and c-Myc protein was also observed in the cell clone (Fig. 1B, 1C, and 1D). These results indicate that we successfully obtained a cell clone in which expression of four factors could be regulated by DOX. This cell clone was used in all later parts of the present study.

\section{Induced expression of OSKM remodeled the phenotype of the cells in vitro}

To test if induced OSKM expression could change the phenotype of the cells from melanoma cells to melanoma stem cells, the cell sphere formation culture technique, which has been broadly used to estimate the numbers of CSCs were used, combined with the addition of basic fibroblast growth factor (bFGF) which helps to maintain the "stemness" of the cells in culture. The cells were cultured in the presence or absence of DOX. Fig. 2A shows representative microscopic appearances. Fig. 2B shows the statistically analyzed results. The cell sphere numbers were significantly increased in the cells cultured with DOX, compare with the cells without DOX treatment (Fig. 2A and 2B). These results indicate that the induction of OSKM in melanoma cells by DOX increased the number of tumor initiating cells and 
suggest that the induced expression of the four factors could remodel the phenotype of the cells from melanoma cells into MSCs in vitro.

\section{Induced expression of OSKM up-regulated stat3 expression}

In order to explore the underlying mechanism, by which OSKM remodels the phenotype of the cells, the mRNAs of related genes were analyzed with RT-QPCR. These genes include signal transducer and activator of transcription 3 (Stat3), Cyclin-A1, cyclin dependent kinase 1 (CDK1), S-phase kinase-associated protein 2 (SKP2) and transformation related protein 53 (Trp53, P53). Stat3 has been reported to maintain the stemness of radial glia at mid-neurogenesis [21]; to enhance the in vitro self-renewal and in vivo repopulating activities of hematopoietic stem cells [22] and to enhance stemness in human pancreatic cancer [23]. Downregulation of
Cyclin B1/CDK1 signaling has been reported being involved in inducing $\mathrm{G} 2 / \mathrm{M}$ arrest and suppressing tumorigenesis of renal cell carcinoma [24]. It has been reported that the beta-TrCP-FBXW2-SKP2 axis forms an oncogene-tumour suppressor-oncogene cascade to control cancer cell growth [25]. p53 has been recognized as a promising molecular target in therapeutic perspectives of malignant tumors with an emphasis on clinical trial results to date [26]. The results are shown in Table 2. Among all analyzed mRNAs, only the mRNA of Stat3 was significantly increased after exposure of the cells to DOX. All the other parameters were unchanged by DOX. Based on the basal function of Stat3 in maintaining "stemness" of normal and malignant stem cells, it is reasonable to postulate that increased expression of Stat 3 might be responsible for remodeling of the cell phenotype.
A

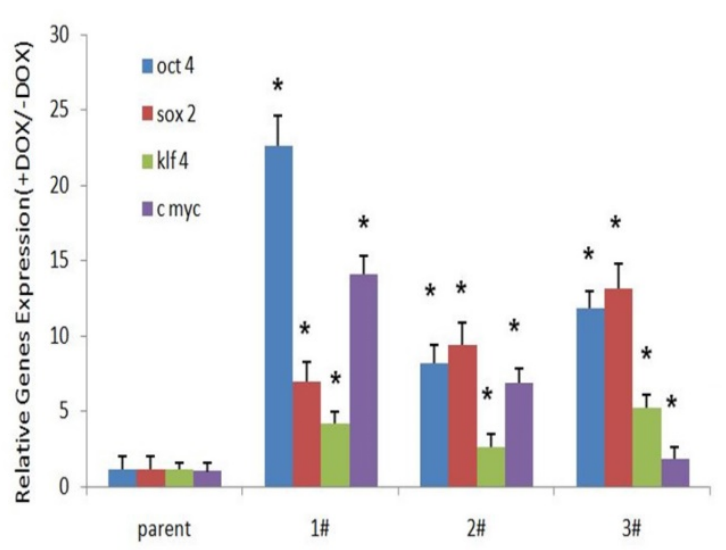

C

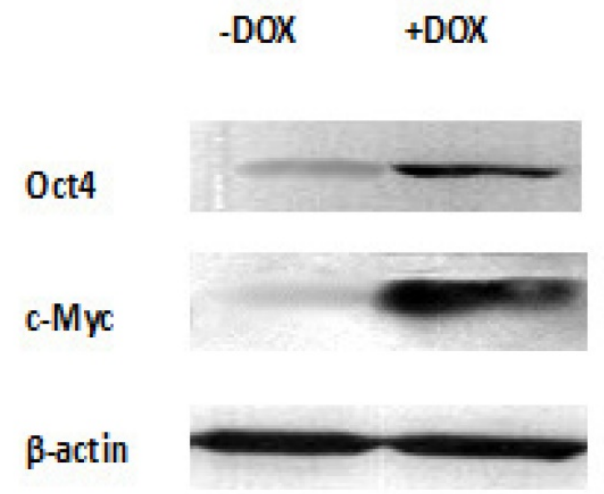

B

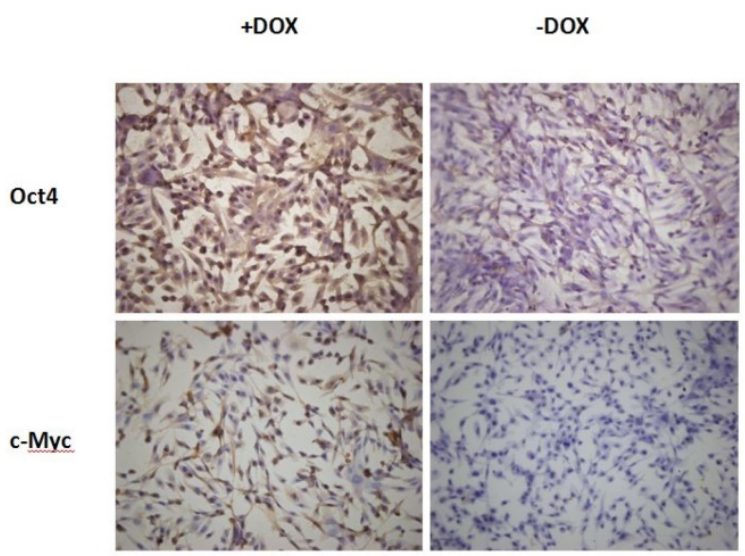

D

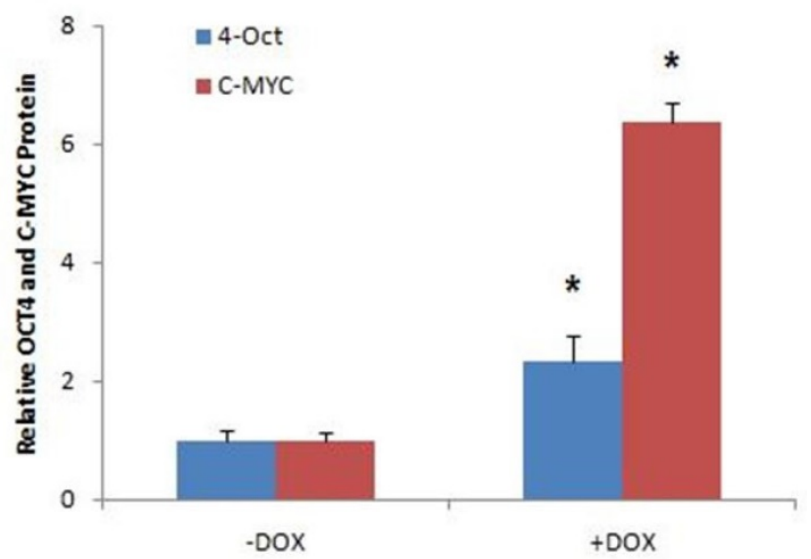

Figure 1. Induction of OSKM by DOX treatment Cell clones, transfected with TetO-FUW-OSKM, were cultured in the presence or absence of DOX. The mRNAs and proteins were analyzed by RT-QPCR (A), immunochemistry (B) and western blot (C and D), respectively. The amount of mRNAs and proteins were normalized to beta-actin mRNA or protein. Relative fold activation was obtained based on the ratio of the normalized values of each group cells induced by DOX to that of the cells without exposure to DOX (A and $D)$. The data are expressed as the mean $\pm S D, N=6$, *P $<0.01$ versus DOX (-) 
A

-DOX

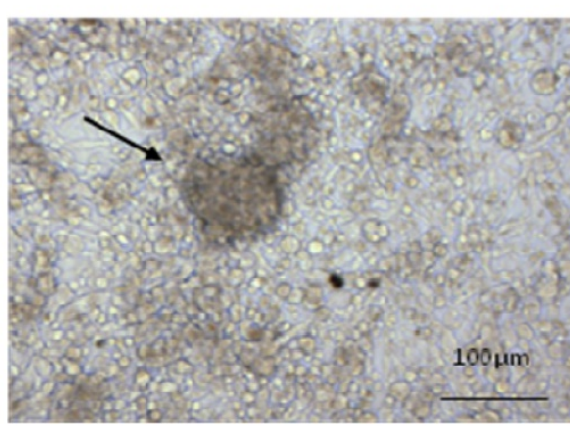

B
$+\mathrm{DOX}$

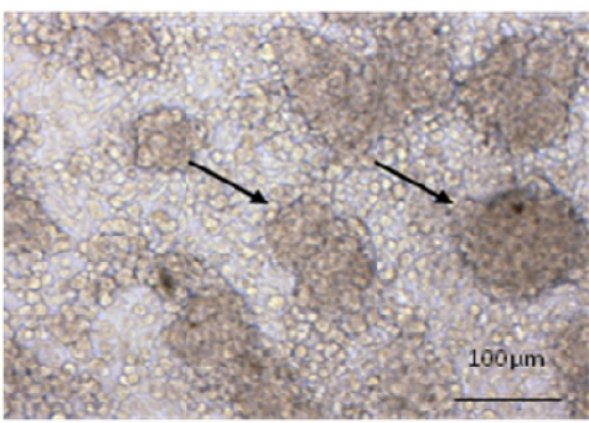

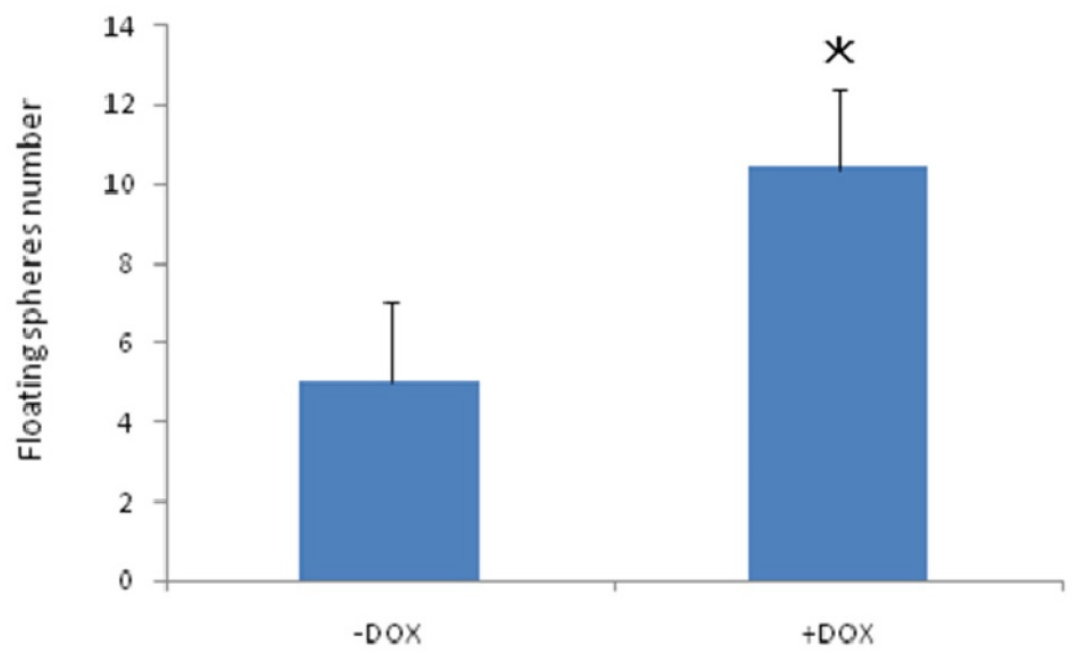

Figure 2. Induction of OSKM increased the number of tumor initiating cells in vitro Cell sphere formation culture was used to estimate the numbers of CSCs in the presence or absence of DOX. A: representative microscopic appearances and B: the statistically analyzed results. The data were expressed as the mean $\pm S D, N=6, * P<0.05$ versus DOX (-).

Table 2. Effects of the induction of OSKM on the expression of related genes.

\begin{tabular}{llllll}
\hline & Stat3 & CyclinA1 & CDK1 & SKP2 \\
\hline -DOX & 1.00 & 1.00 & 1.00 & 1.00 & 1.00 \\
+ DOX & $4.30 \pm 0.42^{* *}$ & $1.11 \pm 0.10$ & $0.90 \pm 0.07$ & $1.22 \pm 0.05$ & $1.39 \pm 0.17$
\end{tabular}

The mRNAs were analyzed by RT-QPCR and were normalized to $\beta$-actin mRNA. Relative fold activation was calculated based on the ratio of the normalized values of the cells induced by DOX to that of the cells without exposure to DOX. The data are expressed as the mean $\pm \mathrm{SD}, \mathrm{N}=6,{ }^{* *} \mathrm{P}<0.01$, versus DOX (-).

\section{Induced expression of OSKM remodeled the phenotype of the cells in vivo}

In order to determine if the induced expression of OSKM can remodel the phenotype of the cells, in vivo, B16-F10 melanoma cells were injected into C57BL/6 mice, to generate subcutaneous tumors. Drinking water with or without addition of DOX was given to the mice. After two weeks, the mice were sacrificed to obtain the subcutaneous tumor. Volume and weight of the tumors were measured. Fig. 3A shows specimens of the subcutaneous tumors and Fig. 3B shows statistically analyzed results. The average volume and weight of the subcutaneous tumors, from the mice receiving DOX, were significantly greater than those from the mice not receiving DOX (Fig. 3B), suggesting that the induction of OSKM by DOX also increased the number of tumor-initiating cells in vivo. These results verify the conclusion that the induced expression of the four factors could remodeled the phenotype of the cells from melanoma cells into MSCs in vivo also. 
A

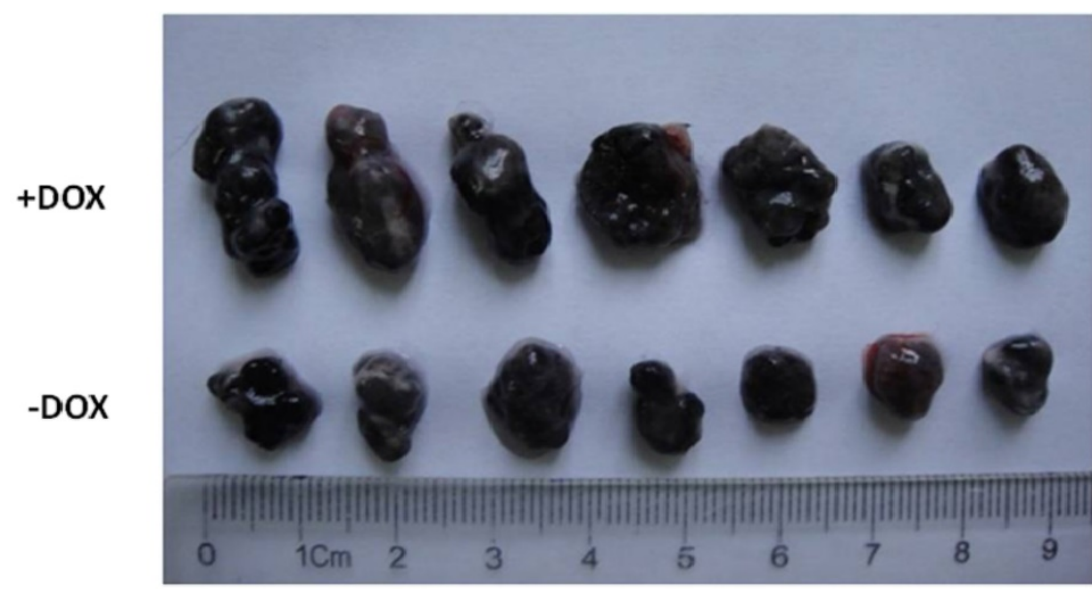

B

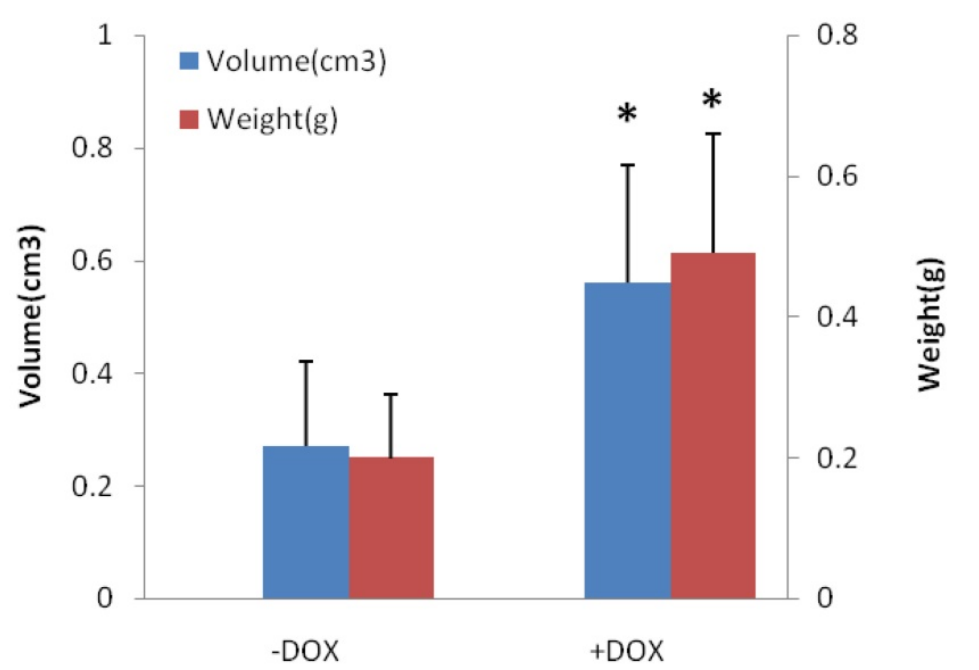

Figure 3. Induction of OSKM increased the numbers of tumor initiating cells in vivo The cells were injected into C57BL/6 mice subcutaneously and drinking water with or without addition of DOX was supplied. The mice were sacrificed and subcutaneous tumors were taken. The volume and weight of the tumors were measured. A: general specimen of subcutaneous tumors and $\mathrm{B}$ : statistically analyzed results. The data are expressed as the mean $\pm \mathrm{SD}, \mathrm{N}=6,{ }^{\mathrm{P}}<0.05$ versus DOX (-).

\section{Discussion}

The limited availability of MSCs, which play a crucial role in melanoma recurrence, relapse, and drug resistance, and are therefore a promising target for novel therapeutic reagents [9-14], restricts the potential for studies on molecular mechanisms and anti tumor therapeutic reagent screening [27]. In the present study, mouse melanoma F10-B16 cells were co-transfected with the plasmids TetO-FUW-OSKM and FUW-M2rtTA, which have been used to reprogram somatic cells and as a drug-inducible system [28, 29]. As expected, in all analyzed three stable cell clones, the mRNAs of Oct4, Sox2, Klf4 and c-Myc (and, in one stable cell clone), the protein expression of Oct4 and c-Myc were highly induced after treatment with DOX. The result is supported by the knowledge that plasmid TetO-FUW-OSKM is a single polycistronic vector encoding the four transcription factors driven by Tet-On element and indicates that we have successfully obtained the cell clone in which expression of four factors could be regulated by DOX. The result provided the preliminary basis for the next part of this study.

Tumor initiating ability has been accepted as characteristics of cancer stem cells in the field [5]. In the present study, we found that the induced expression of the four transcription factors in melanoma F10-B16 cells significantly increased the number of tumor initiating cells in the cultured cell population. We concluded that the phenotypic remodeling of the cells from melanoma into its stem 
cells had happened due to induced expression of these factors. This conclusion was further supported by the observation that these four factors also increased the number of tumor initiating cells of melanoma cells in vivo in the present study. It also corroborates data from other published studies showing that these factors remodel the cell phenotype of osteosarcoma and breast cancer cells into osteosarcoma stem cells and breast cancer stem cells respectively [16-18].

The expression of particular genes determines the characteristic phenotype and the function of the cells. To explore the molecular mechanism by which the induced expression of these four factors affected the phenotype of the melanoma cells, the mRNA level of the related genes was analyzed. We paid special attention to the genes, whose functions have been involved in maintaining stem cell characteristics, cell cycle regulation, cell proliferation and apoptosis. We found that among all analyzed mRNAs, exclusively the mRNA of signal transducer and activator of transcription 3 (Stat3), was significantly increased due to induced expression of the four factors and all the others, including those who control cell cycle progression and those who play roles in cell proliferation and apoptosis, were unchanged. This result once more supports our argument that the phenotypic remodeling of the cells from melanoma into its stem cells had happened due to induced expression of the four factors, because that function of Stat 3 has been reported to be associated with stem cell maintenance of normal [21, 22] and malignant cells [23]. It is unknown either how these factors, used in the present study, enhance stat 3 expression or which one of them plays a crucial role in the activation. However, recent study has shown that Oct4 or Klf4 alone, but not Sox2, can successfully reprogram fibroblasts into induced pluripotent stem cells with the help of epithelial cell adhesion molecule (a trans-membrane protein) and its extracellular domain (a soluble protein), via activation of Stat3, which leads to the nuclear-translocation of HIF2a [30]. This study provides an evidence to show that Oct4 and Klf4 of the four factors may play a crucial role in activation of Stat 3 and again supports our above argument. The result further reveals that the Stat 3 might be a crucial link between the induction of four factors and the cell remodeling. It indicates that Stat3 up-regulation might play an important role in sustaining the phenotype of MSCs, suggesting it might serve as the potential target against MSCs to overcome malignant melanoma. Present study could not answer a detail molecular mechanism on how Stat 3 change naive melanoma towards a tumor-initiating cell phenotype. Furthermore, there is no direct report found from published literature on it until now. However, a study has shown that Stat 3 changes naive melanoma to a melanoma-initiating cell phenotype, by up-regulation of FN1, SNAIL, OCT4 and NANOG, that could favor chemotherapy resistance and relapse [31]

\section{Conclusions}

In the present study, we found that induced expression of four stem cell factors remodeled the phenotype of melanoma cells. This conclusion was supported by increasing the numbers of melanoma stem cells in both in vitro and in vivo experimental models and by exclusively increasing the expression of stat3. The phenotypic remodeling of melanoma cells following the induction of these four factors provided a simple and optimal means to constantly obtain melanoma stem cells for screening new therapeutic reagents. The result also reveals that Stat3 may be a crucial link between the induction of the four factors and the cell remodeling, suggesting its potential role as a target to fight melanoma.

\section{Abbreviations}

MSCs: melanoma stem cells, TICs: tumor-initiating cells, CSCs: cancer stem cells, OSKM: Yamanaka's four transcription factors, Oct4, Sox2, Klf4, and c-Myc, Oct4: octamer-binding transcription factor-4, Sox2: SRY-related high-mobility-group (HMG)-box protein-2, Klf4: Kruppel-like factor-4, c-Myc: MYC proto-oncogene, DOX: doxycycline, Stat3: signal transducer and activator of transcription 3, SKP2: S-phase kinase-associated protein 2, P53: transformation related protein 53, FN1: fibronectin 1, SNAIL: transcriptional factor snail, NANOG: Nanog homeobox.

\section{Acknowledgments}

This study was supported by the National Natural Science Foundation of China (Grants: NSFC No. 21277057). We would like to express our great appreciation to Professor F. William Orr from the University of Manitoba in Canada for his great help in revising the manuscript and to Professor Rudolf Jaenisch from the Whitehead Institute for Biomedical Research in USA for his great help in contributing the plasmids FUW-M2rtTA and TetO-FUW-OCT4 to Addgene.

\section{Competing Interests}

The authors have declared that no competing interest exists.

\section{References}

1. DeSantis CE, Lin CC, Mariotto AB, Siegel RL, Stein KD, Kramer JL, et al. Cancer treatment and survivorship statistics, 2014. CA: a cancer journal for clinicians. 2014; 64: 252-71. 
2. Hoang MT, Eichenfield LF. The rising incidence of melanoma in children and adolescents. Dermatology nursing. 2000; 12: 188-9, 92-3.

3. Dudek-Peric AM, Ferreira GB, Muchowicz A, Wouters J, Prada N, Martin S, et al. Antitumor immunity triggered by melphalan is potentiated by melanoma cell surface-associated calreticulin. Cancer research. 2015; 75: 1603-14.

4. Ribas A, Hamid O, Daud A, Hodi FS, Wolchok JD, Kefford R, et al. Association of Pembrolizumab With Tumor Response and Survival Among Patients With Advanced Melanoma. Jama. 2016; 315: 1600-9.

5. Reya T, Morrison SJ, Clarke MF, Weissman IL. Stem cells, cancer, and cancer stem cells. Nature. 2001; 414: 105-11.

6. Singh A, Settleman J. EMT, cancer stem cells and drug resistance: an emerging axis of evil in the war on cancer. Oncogene. 2010; 29: 4741-51.

7. Kolev VN, Wright QG, Vidal CM, Ring JE, Shapiro IM, Ricono J, et al. $\mathrm{PI} 3 \mathrm{~K} / \mathrm{mTOR}$ dual inhibitor VS-5584 preferentially targets cancer stem cells. Cancer research. 2015; 75: 446-55

8. Satapathy SR, Siddharth S, Das D, Nayak A, Kundu CN. Enhancement of Cytotoxicity and Inhibition of Angiogenesis in Oral Cancer Stem Cells by a Hybrid Nanoparticle of Bioactive Quinacrine and Silver: Implication of Base Excision Repair Cascade. Molecular pharmaceutics. 2015; 12: 4011-25.

9. Fang D, Nguyen TK, Leishear K, Finko R, Kulp AN, Hotz S, et al. A tumorigenic subpopulation with stem cell properties in melanomas. Cancer research. 2005; 65: 9328-37.

10. Schatton T, Murphy GF, Frank NY, Yamaura K, Waaga-Gasser AM, Gasser M, et al. Identification of cells initiating human melanomas. Nature. 2008; 451: 345-9.

11. Liu S, Kumar SM, Martin JS, Yang R, Xu X. Snail1 mediates hypoxia-induced melanoma progression. The American journal of pathology. 2011; 179: 3020-31.

12. Monzani E, Facchetti F, Galmozzi E, Corsini E, Benetti A, Cavazzin C, et al. Melanoma contains CD133 and ABCG2 positive cells with enhanced tumourigenic potential. European journal of cancer. 2007; 43: 935-46.

13. Civenni G, Walter A, Kobert N, Mihic-Probst D, Zipser M, Belloni B, et al. Human CD271-positive melanoma stem cells associated with metastasis establish tumor heterogeneity and long-term growth. Cancer research. 2011; 71: 3098-109.

14. Luo Y, Dallaglio K, Chen Y, Robinson WA, Robinson SE, McCarter MD, et al. ALDH1A isozymes are markers of human melanoma stem cells and potential therapeutic targets. Stem cells. 2012; 30: 2100-13.

15. Takahashi K, Yamanaka S. Induction of pluripotent stem cells from mouse embryonic and adult fibroblast cultures by defined factors. Cell. 2006; 126: 663-76.

16. Levings PP, McGarry SV, Currie TP, Nickerson DM, McClellan S, Ghivizzani $\mathrm{SC}$, et al. Expression of an exogenous human Oct-4 promoter identifies tumor-initiating cells in osteosarcoma. Cancer research. 2009; 69: 5648-55.

17. Beltran AS, Rivenbark AG, Richardson BT, Yuan X, Quian H, Hunt JP, et al. Generation of tumor-initiating cells by exogenous delivery of OCT4 transcription factor. Breast cancer research : BCR. 2011; 13: R94.

18. Corominas-Faja B, Cufi S, Oliveras-Ferraros C, Cuyas E, Lopez-Bonet E, Lupu $\mathrm{R}$, et al. Nuclear reprogramming of luminal-like breast cancer cells generates Sox2-overexpressing cancer stem-like cellular states harboring transcriptional activation of the mTOR pathway. Cell cycle. 2013; 12: 3109-24.

19. Wang Y, Wei Y, Zhang H, Shi Y, Li Y, Li R. Arsenic trioxide induces apoptosis of p53 null osteosarcoma MG63 cells through the inhibition of catalase. Medical oncology. 2012; 29: 1328-34.

20. Yi L, Zhou ZH, Ping YF, Chen JH, Yao XH, Feng H, et al. Isolation and characterization of stem cell-like precursor cells from primary human anaplastic oligoastrocytoma. Modern pathology : an official journal of the United States and Canadian Academy of Pathology, Inc. 2007; 20: 1061-8.

21. Hong S, Song MR. Signal transducer and activator of transcription-3 maintains the stemness of radial glia at mid-neurogenesis. J Neurosci. 2015; 35: 1011-23.

22. Hong SH, Yang SJ, Kim TM, Shim JS, Lee HS, Lee GY, et al. Molecular integration of HoxB4 and STAT3 for self-renewal of hematopoietic stem cells: a model of molecular convergence for stemness. Stem Cells. 2014; 32: 1313-22.

23. Panni RZ, Sanford DE, Belt BA, Mitchem JB, Worley LA, Goetz BD, et al. Tumor-induced STAT3 activation in monocytic myeloid-derived suppressor cells enhances stemness and mesenchymal properties in human pancreatic cancer. Cancer Immunol Immunother. 2014; 63: 513-28.

24. Pan XW, Chen L, Hong Y, Xu DF, Liu X, Li L, et al. EIF3D silencing suppresses renal cell carcinoma tumorigenesis via inducing $\mathrm{G} 2 / \mathrm{M}$ arrest through downregulation of Cyclin B1/CDK1 signaling. Int J Oncol. 2016; 48: 2580-90.

25. Xu J, Zhou W, Yang F, Chen G, Li H, Zhao Y, et al. The beta-TrCP-FBXW2-SKP2 axis regulates lung cancer cell growth with FBXW2 acting as a tumour suppressor. Nat Commun. 2017; 8: 14002

26. Ferraz da Costa DC, Fialho E, Silva JL. Cancer Chemoprevention by Resveratrol: The p53 Tumor Suppressor Protein as a Promising Molecular Target. Molecules. 2017; 22.

27. Kumar D, Gorain M, Kundu G, Kundu GC. Therapeutic implications of cellular and molecular biology of cancer stem cells in melanoma. Molecular cancer. 2017; $16: 7$

28. Hockemeyer D, Soldner F, Cook EG, Gao Q, Mitalipova M, Jaenisch R A drug-inducible system for direct reprogramming of human somatic cells to pluripotency. Cell stem cell. 2008; 3: 346-53.

29. Carey BW, Markoulaki S, Hanna J, Saha K, Gao Q Mitalipova M, et al. Reprogramming of murine and human somatic cells using a single polycistronic vector. Proceedings of the National Academy of Sciences of the United States of America. 2009; 106: 157-62.
30. Kuan, II, Liang KH, Wang YP, Kuo TW, Meir YJ, Wu SC, et al. EpEX/EpCAM and Oct 4 or Klf4 alone are sufficient to generate induced pluripotent stem cells through STAT3 and HIF2alpha. Scientific reports. 2017; 7: 41852.

31. Ohanna M, Cheli Y, Bonet C, Bonazzi VF, Allegra M, Giuliano S, et al. Secretome from senescent melanoma engages the STAT3 pathway to favor reprogramming of naive melanoma towards a tumor-initiating cell phenotype. Oncotarget. 2013; 4: 2212-24. 